\title{
Wavelength Division Demultiplexing and Cross-Talk Assessment of a Photonic Crystal Filter
}

\author{
K. Lenglé, M. Gay, L. Bramerie, J. C. Simon, S. Combrié, G. Lehoucq, A. de Rossi, S. Malaguti, \\ G. Bellanca, S. Trillo
}

\begin{abstract}
We report on the design, fabrication and characterization of a filter for demultiplexing in the wavelength division domain realized with a $\mathrm{HO}$ cavity on a III-V photonic crystal $(\mathrm{PhC})$ membrane. This device is single mode, the total insertion losses are about $10.5 \mathrm{~dB}$, the drop efficiency is close to $40 \%$ and the channel isolation can exceed $20 \mathrm{~dB}$ at $500 \mathrm{GHz}$. $28 \mathrm{Gbit} / \mathrm{s} \mathrm{NRZ}$ wavelength demultiplexing shows less than $0.5 \mathrm{~dB}$ penalties. Cross talk measurements demonstrate less than $1 \mathrm{~dB}$ penalty when adjacent channels are spaced as closely as $235 \mathrm{GHz}$, demonstrating the good compatibility of this filter with LAN-WDM systems. Finally, $56 \mathrm{Gbit} / \mathrm{s}$ NRZ-DQPSK signal filtering is assessed revealing negligible penalty $(<0.5 \mathrm{~dB})$.
\end{abstract}

Index Terms-Photonic integrated circuits, optical filters, photonic crystal waveguide, optical signal processing, wavelength division demultiplexing.

\section{INTRODUCTION}

Over the last few years, photonic crystals $(\mathrm{PhC})$ have been demonstrated as promising candidates for large integration scale devices for all-optical communication networks [1]-[4]. Extremely compact PhC microcavity filters for optical communications have been proposed and demonstrated in the literature, with promising results, such as add-drop filters [5], channel drop filters [6]-[8], bandstop filters [9] or bandpass filters [10]. In this letter system experiments are performed for the first time on a III-V PhC drop filter for channel demultiplexing, demonstrating the maturity of the device: its filtering ability including cross talk measurements with a $28 \mathrm{Gbit} / \mathrm{s}$ NRZ signals is explored. Finally the potential of the filter at 56 Gbit/s NRZ-DQPSK is assessed.

Manuscript received $5^{\text {th }}$ of July 2012. This work was supported in part by European Union, FP7/ICT funding programme, through the Copernicus project (249012) (www.copernicusproject.eu) and by Region Bretagne.

K. Lenglé, M. Gay, L. Bramerie and J-C. Simon are with Université Europenne de Bretagne (UEB), CNRS-Foton Laboratory (UMR 6082) Université de Rennes 1, Enssat, BP 80518, 22305 Lannion Cedex, France (+33296469206; fax: +33296370199; e-mail: lengle@enssat.fr).

S. Combrié, G. Lehoucq, A. de Rossi are with Thales Research and Technology, 1 Avenue A. Fresnel, 91767 Palaiseau, France (e-mail: alfredo.derossi@thalesgroup.com ).

G. Bellanca, S. Malaguti, S. Trillo are with University of Ferrara, Via Saragat 1, 44122 Ferrara, Italy (e-mail: stefania.malaguti@unife.it).

\section{The Photonic Crystal Technology}

The device is a PhC chip based on III-V technology that enables the future integration of light emitting devices, with the potential of ultra-low power consumption, as demonstrated by the NTT group [11]. A processing quality comparable to silicon has been demonstrated on this platform, where cavities with a Q-factor exceeding one million have been achieved [12].

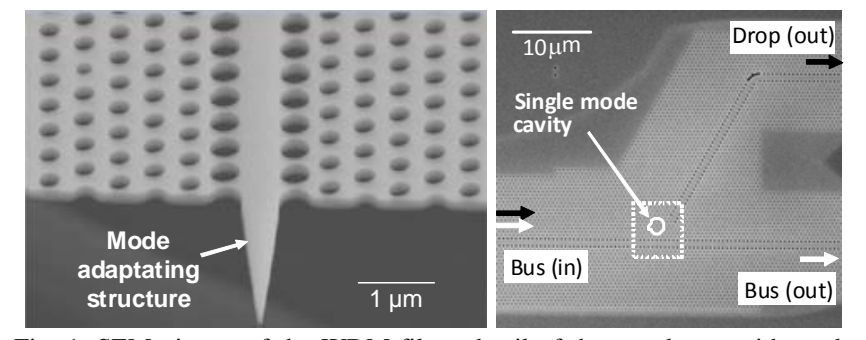

Fig. 1. SEM picture of the WDM filter: detail of the membrane with mode adapter structure (left), filter with the drop channel (right).

Figure 1 shows SEM pictures of the 3-port filter. The structure is an air-suspended GaAs membrane with a 2D triangular-lattice of holes, as shown on the left-hand side of the figure. The waveguide is produced by a single line defect. The filter uses a H0 cavity [13] (highlighted by the circle in the picture on the right) obtained by shifting two adjacent holes of the $\mathrm{PhC}$ crystal structure. The analysis and the design of the filters are first based on coupled mode theory (CMT) [14]. The parameter specified with the help of CMT (coupling strength, and frequency of the resonance) are translated into a $\mathrm{PhC}$ cavity design using a home-made 3D FDTD running on a small scale $(<50)$ cluster of processors. Cavities have been optimized individually. Non linear effects were neglected as an average power of a few $\mathrm{mW}$ range was considered (which is appropriate to the filtering of a WDM signal). The fabrication is detailed in [12] and is based on e-beam lithography, inductively coupled plasma etching and subsequent removal of the sacrificial layer (GaAs) such that a self-standing membrane on GaInP is left. As reported in [15], for a single cavity based filter, a maximum drop efficiency of $50 \%$ can be theoretically reached. In this configuration, high drop efficiency is pursued by adjusting the transmission properties of the bus and drop waveguides and the cavity to 
waveguide coupling strength, respectively. Optimizations can be obtained by correctly positioning the cavity with respect to the bus and drop waveguides and by modifying the radius of the holes in the two rows adjacent to the waveguide.

A $60^{\circ}$ bend has been introduced on the drop waveguide, to align the drop port with the bus one, thus facilitating both measurements and final pigtailing. 3D-FDTD was also used for this optimization revealing a transmission of more than $95 \%$ in the band of interest.

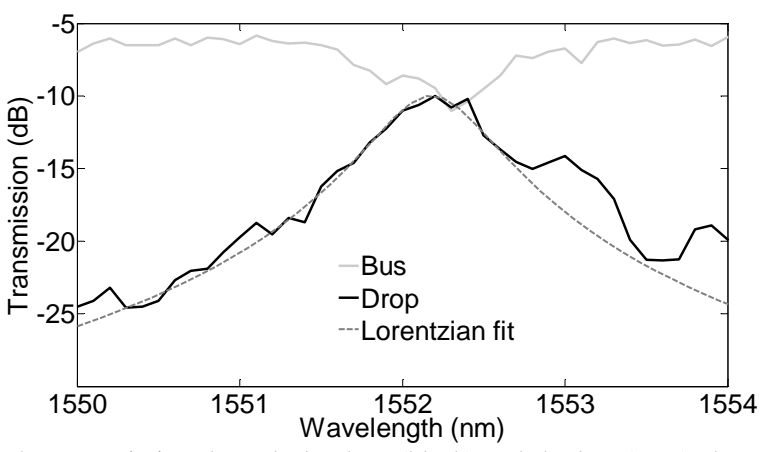

Fig. 2. Transmission through the drop (black) and the bus (grey) channels. The dashed curve corresponds to the Lorentzian fit of the drop channel.

In order to reduce the total insertion losses and decrease Fabry-Perot oscillations, we have improved the fiber to $\mathrm{PhC}$ waveguide coupling through an integrated mode adapters [16] (see Fig. 1 on the left). By using microlensed fibers providing a focus spot of $2 \mu \mathrm{m}$ at $1 / \mathrm{e}^{2}$ intensity, losses of about $2.5 \mathrm{~dB}$ per face were obtained in TE mode, a record with such small modes. For this filter, the total insertion losses at the drop port from fiber to fiber (thus including fiber to $\mathrm{PhC}$ input and output coupling loss, propagation loss and the intrinsic drop efficiency) are of about $10.5 \mathrm{~dB}$. Total insertion losses for the bus channel were measured to be as low as $6 \mathrm{~dB}$.

Fig. 2 shows the transmission curves of bus and drop ports. The $3 \mathrm{~dB}$ bandwidth of the filter is of $0.8 \mathrm{~nm}$ and has been measured by fitting the transmission curve of the drop port with a Lorentzian function, as expected from theory. The drop efficiency is commonly defined as the ratio of the power droped by the cavity from the bus to the drop channel (immediately before and after the cavity) [15]. This parameter is estimated here from the total loss, the coupling losses and the waveguide propagation loss. The estimated waveguide loss is $1 \mathrm{~dB} / \mathrm{mm}$ [16]. The estimated drop efficiency of this filter is thus $38 \%$.

Moreover, we observe that $500 \mathrm{GHz}$ away from the resonance, the signal through the drop is below $20 \mathrm{~dB}$, thus highlighting the good selectivity of the filter.

\section{SYSTEM ASSESSMENT}

\section{A. Back to back experiment at $28 \mathrm{Gbit} / \mathrm{s}$}

In a first experiment, we evaluate the possibility of transmitting a $28 \mathrm{Gbit} / \mathrm{s}$ NRZ signal into the device in a back to back experiment (with the device under test (DUT) placed in between the transmitter and the receiver). The pseudorandom binary sequence (PRBS) length is $2^{31}-1$ bit.

Figure 3 shows the bit error rate (BER) evolution as a function of the received input power of the reference (grey triangles) and filtered (black triangles) signal. The results show no significant penalty (less than $0.5 \mathrm{~dB}$ ) after passing through the device.

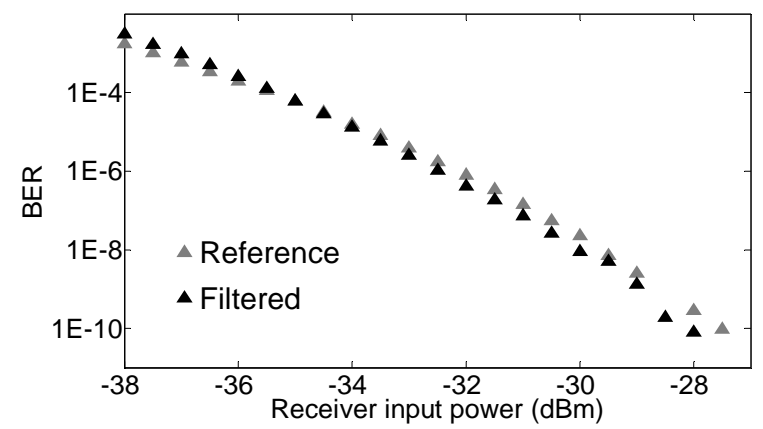

Fig. 3. BER measurements with $28 \mathrm{Gbit} / \mathrm{s}$ NRZ signal through the PhC drop filter.

Additionally, cross talk measurements were performed at $28 \mathrm{Gbit} / \mathrm{s}$ with the NRZ signal. As shown in Fig. 4, three different wavelengths modulated by $28 \mathrm{Gbit} / \mathrm{s}$ NRZ signals were injected into the filter. The channel under study (central wavelength) and the two adjacent channels were modulated by different modulators, in order to obtain uncorrelated signals.

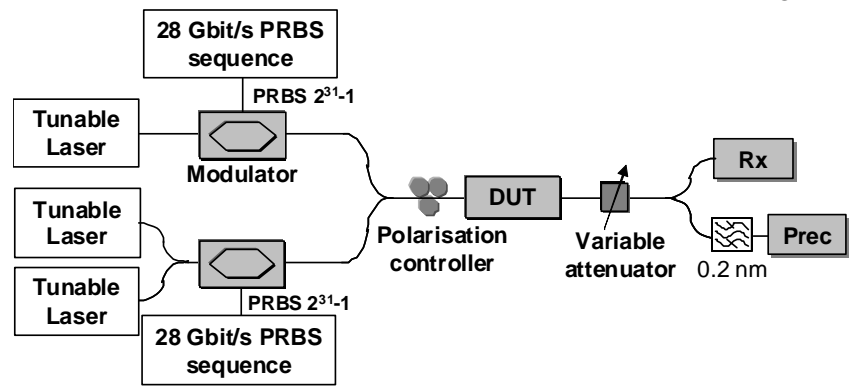

Fig. 4. Experimental setup for the $28 \mathrm{Gbit} / \mathrm{s}$ NRZ cross talk experiment. Polarizations of the three co-polarized signals are controlled and powers are equalised in front of the DUT. Finally a direct detection setup is used for BER measurements. The impact of the adjacent channels on the central one was considered as a function of the channel spacing (frequency shift between signals) and evaluated as the power penalty i.e the increase in the power required to guarantee a BER of $10^{-9}$. In these measurements, the power required for the desired BER with only the central channel on the system was first evaluated and considered as a reference. By measuring the BER as a function of the receiver input power, no error floor was observed from 800 to $200 \mathrm{GHz}$ channel spacing. At $200 \mathrm{GHz}$ spacing, an error floor was measured at a BER of $10^{-10}$.

Fig. 5 summarizes the evolution of the penalty as a function of the channel spacing (black squares). The first observation is that, above $300 \mathrm{GHz}$ spacing, there is no significant penalty induced by the presence of adjacent channels. Now, at $1 \mathrm{~dB}$ penalty at a BER of $10^{-9}$, the channel spacing is $235 \mathrm{GHz}$ showing a good tolerance to the signal frequency.

Hence considering $800 \mathrm{GHz}$ channel spacing (standard for LAN-WDM), the laser frequency tolerance is as high as $565 \mathrm{GHz}$ with only $1 \mathrm{~dB}$ penalty on the WDM system.

Finally, we compared our filter to an ideal Lorentzian one with a $3 \mathrm{~dB}$ bandwidth of $0.8 \mathrm{~nm}$ synthesized with a 
programmable filter (grey crosses in Fig. 5). In this case, the channel spacing is $170 \mathrm{GHz}$ at $1 \mathrm{~dB}$ penalty, which makes it slightly more tolerant than our PhC filter. The difference is probably determined by the asymmetry and the perturbations in the PhC filter transmission curve, which are clearly evident in the black curve of Fig. 2.

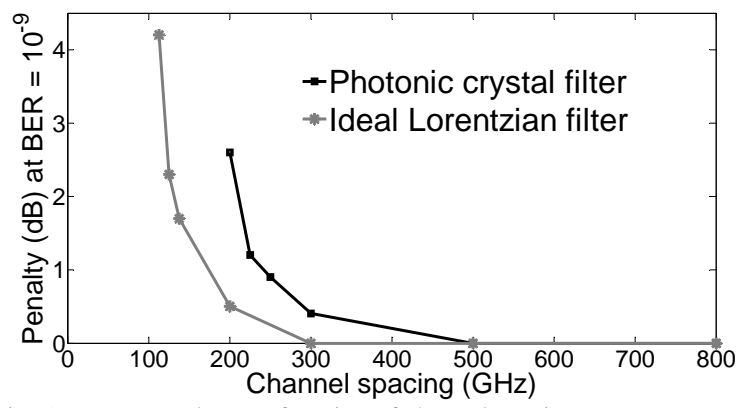

Fig. 5. Power penalty as a function of channel spacing.

\section{B. $56 \mathrm{Gbit/s}$ DQPSK experiment}

The potential of this filter was finally assessed using 56 Gbit/s NRZ-DQPSK format. The experimental setup uses a nested Mach-Zehnder modulator (MZM) with a $2^{15}-1$ bit PRBS length at $28 \mathrm{Gbit} / \mathrm{s}$ at the transmitter. An additional nested MZM was unfortunately not available at the transmitter to do cross talk measurements with DQPSK signal. The preamplified receiver uses a fiber-based delay-line interferometer and balanced detectors before the $28 \mathrm{Gbit} / \mathrm{s}$ receivers for BER analysis. Details of the DQPSK back to back setup can be seen in [17]. The filter was then placed between the transmitter and the receiver in order to assess any degradation the signal could undergo. Fig. 6 shows the BER evolution as a function of the receiver input power for the reference curve (grey symbols) and the filtered signal (black signals) both for in-phase (full symbols) and quadrature phase (empty symbols) output.

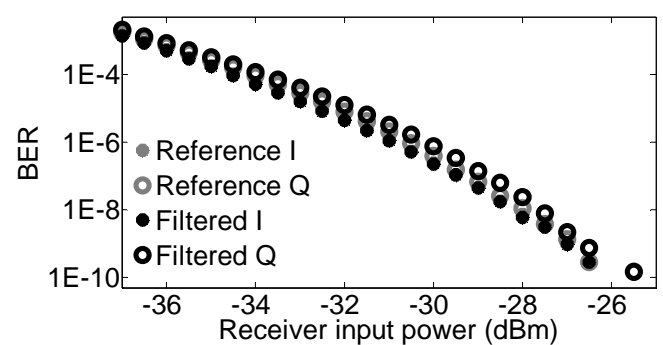

Fig. 6. DQPSK experimental setup.

In both cases penalties are non significant (less than $0.5 \mathrm{~dB}$ ) after passing through the device showing that the device is also compatible with phase modulation formats.

\section{CONCLUSION}

We have developed an optical filter for wavelength demultiplexing, based on III-V semiconductor photonic crystal technology. We report good performance on insertion losses (10.5 dB fiber to fiber), the reaching of a drop efficiency of up to $38 \%$ and a channel isolation of $20 \mathrm{~dB}$ at $500 \mathrm{GHz}$ away from the filter wavelength. The filter is used as a wavelength demultiplexer in a 3 channel, $28 \mathrm{Gbit} / \mathrm{s} \mathrm{NRZ}$ system. No penalty due to the presence of adjacent channels is observed at $800 \mathrm{GHz}$ spacing while a $1 \mathrm{~dB}$ penalty is measured at a BER of $10^{-9}$ with a channel spacing of $235 \mathrm{GHz}$, thus demonstrating that the component is fully compatible with a LAN-WDM context. Finally, no significant penalty $(<0.5 \mathrm{~dB}$ ) after transmission through the filter (on both drop and bus channels) of a $56 \mathrm{Gbit} / \mathrm{s}$ DQPSK signal is measured, showing the potential of such a structure for ultra compact all-optical signal processing. The presented filter is perfectly suited to achieve a multi-channel demultiplexer and this by placing in series several of such filters.

\section{REFERENCES}

[1] K. Nozaki, T. Tanabe, A. Shinya, S. Matsuo, T. Sato, H. Tanitama, M. Notomi, "Sub-femtojoule all-optical switching using a photoniccrystal nanocavity”, Nature Photon., vol. 4, pp. 477-483, 2010.

[2] B. Corcoran, M.D. Pelusi, C. Monat, J. li, L. O’Faolin, T.F. Krauss and B.J. Eggleton, "Ultracompact 160 Gbaud all-optical demultiplexing exploiting slow light in an engineered silicon photonic crystal waveguide”, Opt. Lett., vol. 36, no. 9, pp. 1728-1730, 2011.

[3] P. Colman, C. Husko, S. Combrié, I. Sagnes, C. W. Wong, A. De Rossi, "Observation of soliton pulse compression in photonic crystal waveguides”, Conference postdeadline paper QPDA10, QELS, 2010.

[4] A. Shinya, S. Matsuo, T. Tanabe, E. Kuramochi, T. Sato, M. Notomi, "All-optical on-chip bit memory based on ultra high Q InGaAsP photonic crystal”, Opt. Express, vol. 16, no. 23, pp.19382-19387, 2008.

[5] Z. Qiang, W. Zhou, and Richard A. Soref, “Optical add-drop filters based on photonic crystal ring resonators”, Opt. Express, vol.15, no 4, pp.1823-1831, 2007.

[6] C.C. Wang, L-W. Chen, "Channel drop filters with folded directional couplers in two-dimensional photonic crystals”, Physica B, vol. 405, pp. 1210-1215, 2010.

[7] S. Robinson and R. Nakkeeran, "Channel drop filter based on 2D hetero photonic crystals”, Science, vol.300, pp.1537, 2003.

[8] T. Niemi, L.H. Frandsen, K. Hede, A. Harpoth, P.I. Borel and M. Kristensen, "Wavelength-division demultiplexing using photonic crystal waveguides”, Phot. Techn. Letters, vol. 18, no.1, pp. 226-228, 2006.

[9] F. Monifi, M. Djavid, A. Ghaffari and M. S. Abrishamian, “A new bandstop filter based on photonic crystals”, Proc. PIER, Cambridge, USA, 2008.

[10] C. Chao, X. Li, H. Li, K. Xu, J. Wu and J. Lin, "Bandpass filters based on phase-shifted photonic crystal waveguide gratings”, Opt. Express, vol. 15, no. 18, pp. 11278-11284, 2007..

[11] S. Matsuo, A. Shinya, T. Kakitsuka, K. Nozaki, T.Segawa, T. Sato, Y. Kawaguchi, M. Notomi, "High-speed ultracompactburied heterostrucutre photonic-crystal laser with $13 \mathrm{fJ}$ of energy consumed per bit transmitted”, Nature Photon., vol. 4, pp. 477, 2010.

[12] S. Combrié, Q.V. Tran, C. Husko, P. Colman, and A de Rossi, "High quality GaInP nonlinear photonic crystals with minimized nonlinear absorption“, Appl. Phys. Letters, Vol. 95, pp. 221108, 2009.

[13] Z. Zhang and M. Qiu, "Small-volume waveguide-section high Q microcavities in 2D photonic crystal slabs", Opt. Exp., vol. 12, no.17, pp. 3988-3995, 2004.

[14] C. Manolatou, M. Khan, S. Fan, P. Villeneuve, H. Haus, J. Joannopoulos, "Coupling of modes analysis of resonant channel adddrop filters“, J. Quant. Elec., vol 35, pp 1322-1331, 1999.

[15] H. Ren, C. Jiang, W. Hu, M. Gao, J. Wang,. "Photonic crystal channel drop filter with a wavelength-selective reflection micro-cavity”, Optics Express, vol. 14, no. 6, pp. 2446-2458, 2006..

[16] Q.V. Tran, S. Combrié, P. Colman and A. De Rossi, "Photonic crystal membrane waveguides with low insertion losses”, Appl. Phys. Letters, vol. 95, pp. 061105, 2009.

[17] Y. Ben M'Sallem, Q. T. Le, L. Bramerie, Q. T. Nguyen, E. Borgne, P. Besnard, A. Shen, F. Lelarge, S. La Rochelle, L. A. Rusch, and J. C. Simon, "Quantum-Dash Mode-Locked Laser as a Source for 56Gb/s DQPSK Modulation in WDM Multicast Applications”, Phot. Techn. Letter, v. 23, no. 7, pp.453-455, 2011. 